\title{
Expression of $p 63$ in primary cutaneous adnexal neoplasms and adenocarcinoma metastatic to the skin
}

\author{
Doina Ivan ${ }^{1}$, A Hafeez Diwan ${ }^{1}$ and Victor G Prieto ${ }^{1,2}$ \\ ${ }^{1}$ Department of Pathology and ${ }^{2}$ Department of Dermatology, University of Texas MD Anderson Cancer Center, \\ Houston, TX, USA
}

\begin{abstract}
p63, a recently identified homologue of the p53 gene, has been reported to be essential in the development of epithelia and is mainly expressed by basal and myoepithelial cells. The purpose of this study was to investigate the pattern of p63 expression in cutaneous adnexal neoplasms and to assess its possible value in the differential diagnosis of primary cutaneous neoplasms vs adenocarcinomas metastatic to the skin. Immunohistochemical analysis for p63 was performed on formalin-fixed, paraffin-embedded archival tissue from 20 benign adnexal tumors, 10 malignant adnexal tumors and 14 adenocarcinomas metastatic to the skin. The expression of p63 was evaluated in epidermal cells, skin appendages and metastatic tumor cells. p63 was consistently expressed in the basal and suprabasal cells of epidermis and cutaneous appendages, including the basal/myoepithelial cells of sweat glands. Out of 20 benign adnexal tumors, $13(65 \%)$ showed strong (score 3) p63 expression; the remaining seven (35\%) cases had score 2 . All primary cutaneous carcinomas, including adenocarcinomas, expressed p63. In contrast, none of the metastatic adenocarcinomas to the skin was positive for p63 $(P<0.001)$. Based on our findings, analysis of p63 expression may help in the differential diagnosis of primary vs metastatic cutaneous adenocarcinomas.
\end{abstract}

Modern Pathology (2005) 18, 137-142, advance online publication, 24 September 2004; doi:10.1038/modpathol.3800263

Keywords: $p 63$; cutaneous adnexal neoplasms; metastatic adenocarcinoma to skin

The p63 gene, a recently described member of the p53 gene family, is located on the chromosome 3q27-29. ${ }^{1}$ It contains two separate promoters and expresses at least six major transcripts that lead to two fundamentally different classes of proteins. ${ }^{1-4}$ Three of the $p 63$ isoforms (TAp63) encode proteins with roles similar to p53 (ie transactivation and induction of apoptosis), whereas the other three isoforms $(\Delta \mathrm{N} p 63)$ lack the acidic amino $(\mathrm{N})$-terminal transactivation domain and exert inhibitory effects on p53 activity. It has been recently shown that p63 is highly expressed in the basal cells of human epithelial tissues. ${ }^{5,6}$ Notably, the analysis of RNA from keratinocytes indicated that the major p63 transcripts in these cells encoded $\Delta \mathrm{Np} 63$ isotypes. ${ }^{1}$ Therefore, in these cells the expression of $\Delta \mathrm{Np} 63$ might block the apoptosis-inducing activity of p53

Correspondence: Dr VG Prieto, MD, PhD, Department of Pathology, University of Texas MD Anderson Cancer Center, 1515 Holcombe Boulevard, Unit 85, Houston, TX 77030-4009, USA. E-mail: vprieto@mdanderson.org

Received 17 May 2004; revised and accepted 13 July 2004; published online 24 September 2004 and thus could help maintain the proliferative capacity of basal/progenitor cells. ${ }^{5-8}$

It has been postulated that the p63 plays an essential role in epithelial development, fact demonstrated by the defects or agenesis of squamous epithelia, mammary, lacrimal, salivary glands and craniofacial structures in the p63-deficient mice. ${ }^{7-9}$ In addition, p63 is also expressed in many normal human tissues including prostate basal cells, uterine cervix or urogenital tract. ${ }^{10-13}$ Furthermore, p63 is a selective nuclear marker of the myoepithelial cells in breast and has an increasingly diagnostic value in differentiating between in situ and invasive carcinomas. ${ }^{10,11,14,15}$ Moreover, it has been shown that primary or metastatic carcinomas derived from glandular epithelia, including adenocarcinoma of the breast or prostate, consistently fail to express p63. ${ }^{10,12,14}$

The expression of p63 in normal human epidermis, cutaneous appendages and skin carcinomas has been recently assessed. ${ }^{16,17}$ In these studies, p63 was detected in the epidermal and adnexal basal/ myoepithelial cells and it has been suggested that p63 might be used as a diagnostic marker for epidermal or adnexal tumors in the skin. ${ }^{16-18}$ 
Cutaneous metastases may be the initial manifestation of some neoplasms, including adenocarcinomas. The distinction between such metastases and primary cutaneous neoplasms, especially adnexal tumors, on histologic grounds alone, may be difficult. The purpose of this study was to investigate the pattern of p63 expression in cutaneous adnexal neoplasms, including adenocarcinomas and to assess its possible value in the differential diagnosis of primary cutaneous neoplasms vs adenocarcinomas metastatic to the skin.

\section{Materials and methods}

\section{Patients}

The pathology database at the University of Texas MD Anderson Cancer Center was retrospectively reviewed to identify cases of primary adnexal carcinomas and metastatic adenocarcinomas to the skin between January 1997 and July 2003. There were retrieved 20 benign adnexal tumors (six poromas, three syringomas, nine trichoepitheliomas, one spiradenoma, one papillary eccrine adenoma), 10 malignant adnexal tumors (four microcystic adnexal carcinomas, two eccrine adenocarcinomas, one hidradenocarcinoma, two tricholemmal carcinomas and one mucinous sweat gland adenocarcinoma), and 14 metastatic adenocarcinomas to the skin (12 from breast, two from the gastrointestinal tract). Clinical information was obtained from the medical records. The pathology reports and the hematoxylin and eosin (H\&E)stained glass slides were reviewed in all cases.

\section{Immunohistochemical Staining for p63}

Immunohistochemical analysis for p63 was performed on formalin-fixed, paraffin-embedded archival tissue using the streptavidin-biotin-peroxidase technique. For all cases, a $4 \mu \mathrm{m}$ histologic section was deparaffinized and rehydrated in graded alcohols and distilled water. After blocking the endogenous peroxidase $(3 \%$ hydrogen peroxide for $5 \mathrm{~min}$ ), the heat-induced antigen retrieval was performed in a water bath, using citrate buffer at $\mathrm{pH} 6.0$ for $20 \mathrm{~min}$. Biotin-conjugated secondary antibody was applied for $20 \mathrm{~min}$, and streptavidinbiotin-peroxidase complex (Strept-AB complex, dilution 1:200, DAKO Corp., Carpinteria, CA, USA) was added for 20 min. A mouse antihuman monoclonal antibody that reacts with all p63 isoforms (clone 4A4, dilution 1:200; Santa Cruz, Biotechnology Inc.; Santa Cruz, CA, USA) was used. Positive and negative controls were included in each slide run.

The distribution of the immunoreactivity in the neoplastic cells was analyzed by quantifying nuclear staining, following previous methodology. ${ }^{19}$ A consensus between the investigators was obtained to score the nuclear staining of $p 63$ as follows: 0 for less than $5 \%$ positive nuclei; 1 for $5-25 \%$ positive nuclei, 2 for $26-75 \%$ positive nuclei and 3 for over $75 \%$ positive nuclei. Positivity of cells was defined regardless of staining intensity. By convention, we considered that greater than $25 \%$ positive cells represented the cutoff between negativity and positivity of the p63 immunostaining. Two investigators (VGP and DI) have independently reviewed and scored slides by estimating the percentage of cells exhibiting characteristic nuclear staining. Interobserver variation was addressed by averaging the individual values. The distribution of $p 63$ expression in the basal and suprabasal cells of normal epidermis, hair follicles, sweat glands and sebaceous glands was evaluated in normal skin adjacent to the neoplasms. It was also assessed the pattern of $p 63$ expression in endothelial cells, dermal mesenchymal cells, pilar erector muscles, nerve bundles, adipocytes or inflammatory infiltrates. At least 10 high-power fields were chosen randomly, and 100 cells were counted in each field.

\section{Statistical Analysis}

The statistical association between the distribution of p63 expression in benign or malignant primary cutaneous neoplasms or metastatic adenocarcinomas to the skin was analyzed using $\chi^{2}$ and Fisher's exact tests, with $P<0.05$ considered to be statistically significant.

\section{Results}

Expression of p63 in Normal Epidermis and Skin Appendages

p63 was consistently expressed in the basal and suprabasal cells of normal epidermis; the spinous, granular and corneum layers cells were negative for p63. The outer root sheath cells and the hair bulb of the hair follicle exhibited positivity for $p 63$ whereas the inner root sheath cells and the hair matrix were negative for $p 63$. A variable $p 63$ nuclear staining was identified in the pilar erector muscles. The germinative cells of the secretory portion of the sebaceous glands, as well the duct cells, strongly expressed p63. No immunoreactivity was seen in the mature sebocytes. The myoepithelial cells of the eccrine coils and the basal cells of the eccrine glands ducts were positive for $p 63$. The myoepithelial cells of the secretory component of the apocrine glands and the outer layer cells of the apocrine ducts did also express p63. No differences in the labeling pattern were observed between the epidermis and adnexal structures of the skin. p63 immunostaining was not evident in the nuclei of the endothelial cells, dermal mesenchymal cells, nerves, adipocytes or inflammatory infiltrates. 


\section{Expression of p63 in Benign Adnexal Tumors}

The analysis of $p 63$ expression in trichoepitheliomas (Figure 1a) revealed that $p 63$ was expressed in over $75 \%$ of the basaloid cells (nuclear score 3 ) in six out of nine $(67 \%)$ cases, including desmoplastic trichoepitheliomas. The other three cases were also positive for $p 63$ and had a nuclear score 2. Notably, the latter had areas of $p 63$ negativity alternating with areas of strongly p63 labeling (almost 100\% of the nuclei).

The pattern of expression of p63 in six eccrine poromas (Figure 1b) included in our study revealed that the cords of proliferating basaloid cells were strongly positive (nuclear score 3 ) in three cases and had a nuclear score 2 in the remaining three cases. Only the luminal cells in the tumor nests did not expressed $p 63$.

The myoepithelial cells of the ductal structures of the syringomas (Figure 1c) were positive for p63; two cases had nuclear score 3 and one case, score 2 . The inner layer of cuboidal epithelium did not express $p 63$.

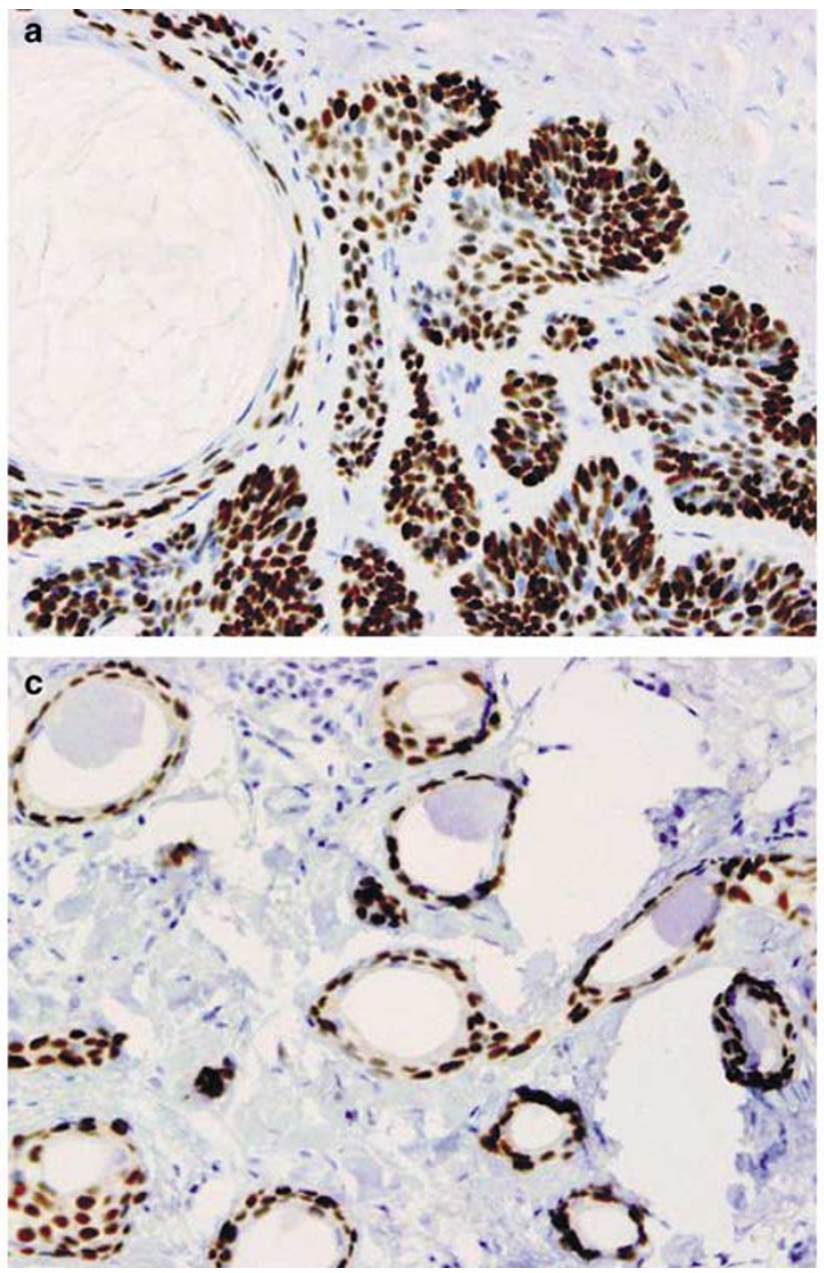

Strong p63 positivity was identified in the outer cells of the basaloid nodules in the eccrine spiradenoma (Figure 1d), whereas the inner cells had a p63 labeling of less than $25 \%$ of the nuclei. The papillary eccrine adenoma case had a similar pattern of p63 immunostaining with strong positivity (nuclear score 3 ) of the outer cells.

\section{Expression of $p 63$ in Malignant Adnexal Tumors}

Both cases of eccrine adenocarcinoma (Figure 2a) were strongly positive (nuclear score 3 ) for p63, as well as the hidradenocarcinoma (Figure 2b). The basaloid islands of the mucinous eccrine adenocarcinoma included in our study expressed p63 in the less than $25 \%$ of cells (nuclear score 1 ).

All four cases of microcystic adnexal carcinoma expressed p63 in more than $75 \%$ of cells (Figure 2c). No differences in the labeling pattern were observed between the superficial and the deeper nests or cords of neoplastic cells. p63 staining highlighted
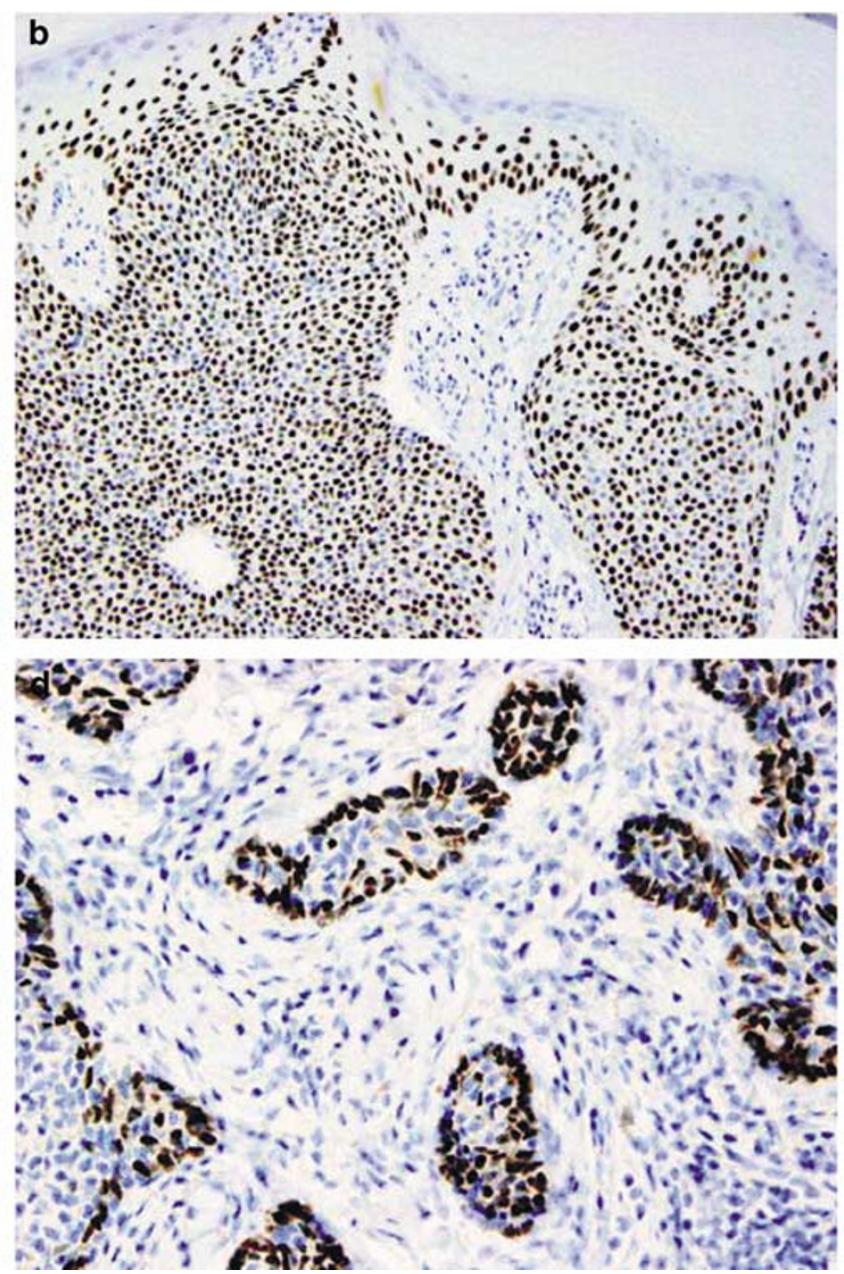

Figure 1 Immunohistochemical staining for p63 reveals strong and diffuse nuclear reactivity in the basaloid cells of trichoepitheliomas (a) and eccrine poromas (b). The myoepithelial cells of the ductal structures of the syringomas expressed p63; the inner layer of cuboidal epithelium did not (c). Also, strong p63 positivity was identified in the outer cells of the basaloid nodules of the eccrine spiradenomas $(\mathbf{d})$. 

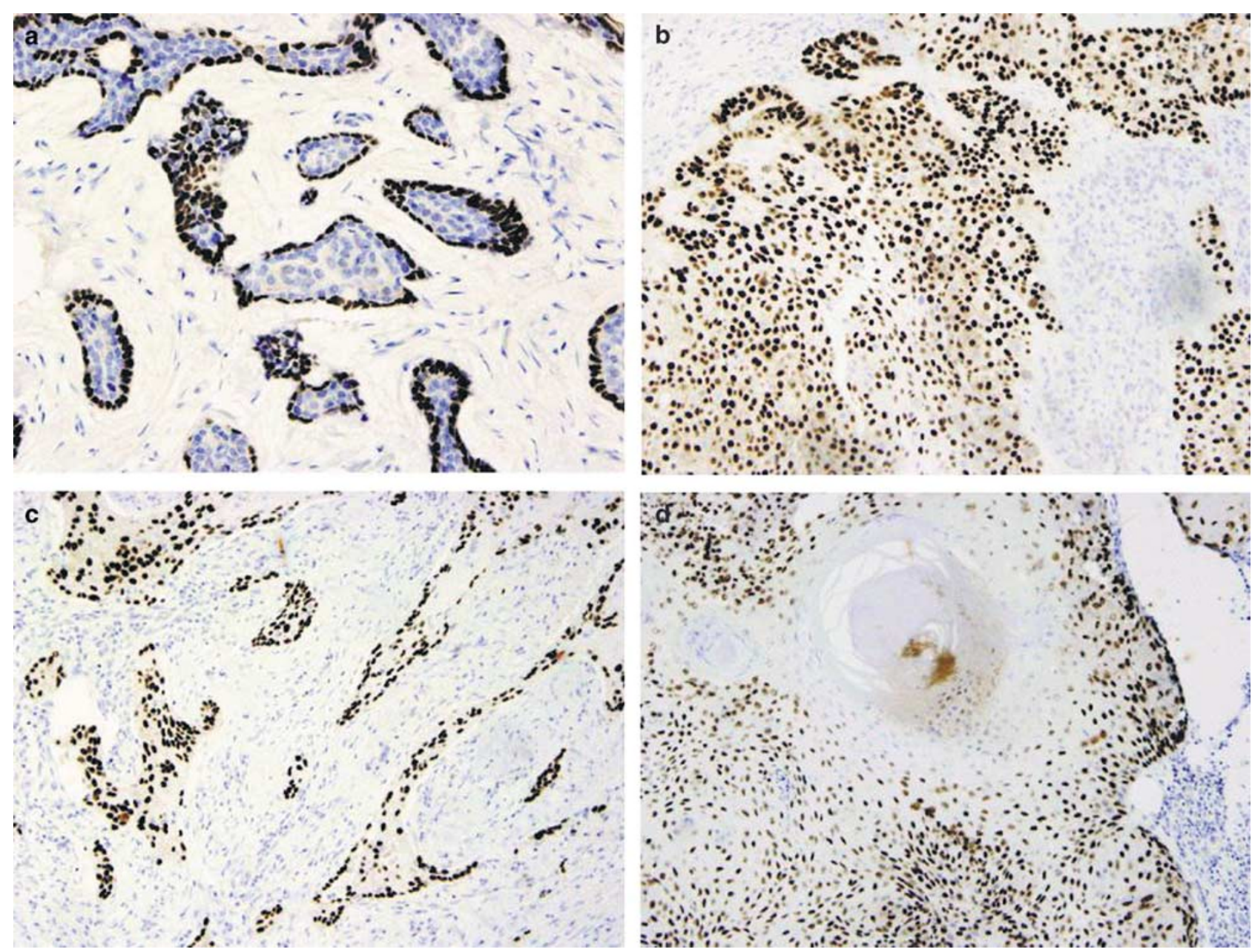

Figure 2 The eccrine adenocarcinomas (a) and the hidradenocarcinoma (b) were strongly positive for p63. The microcystic adnexal carcinomas (c) also expressed p63, as well as the infiltrating lobules of tricholemmal carcinoma (d).

the perineural invasion in one case, since the nerve bundles did not express $p 63$.

The infiltrating lobules of tricholemmal carcinoma (Figure 2d) were also positive for $p 63$ (nuclear score 3).

No cytoplasmic labeling with $p 63$ was observed in any of the primary adnexal carcinomas included in the study.

\section{Expression of p63 in Metastatic Adenocarcinomas to the Skin}

A total of 14 metastatic adenocarcinomas to the skin (12 from breast, two from the gastrointestinal tract) were included in our study and they were all negative for p63 (Figure 3). The majority of the metastatic breast carcinomas did not express p63 in any of the cells. In three $(25 \%)$ of the cases, focal cytoplasmic p63 positivity was identified. The skeletal muscle that was included in some of the studied slides was strongly positive for $p 63$ and the muscle striations were evident.

In the carcinomas metastatic from the gastrointestinal tract (one colonic adenocarcinoma, one

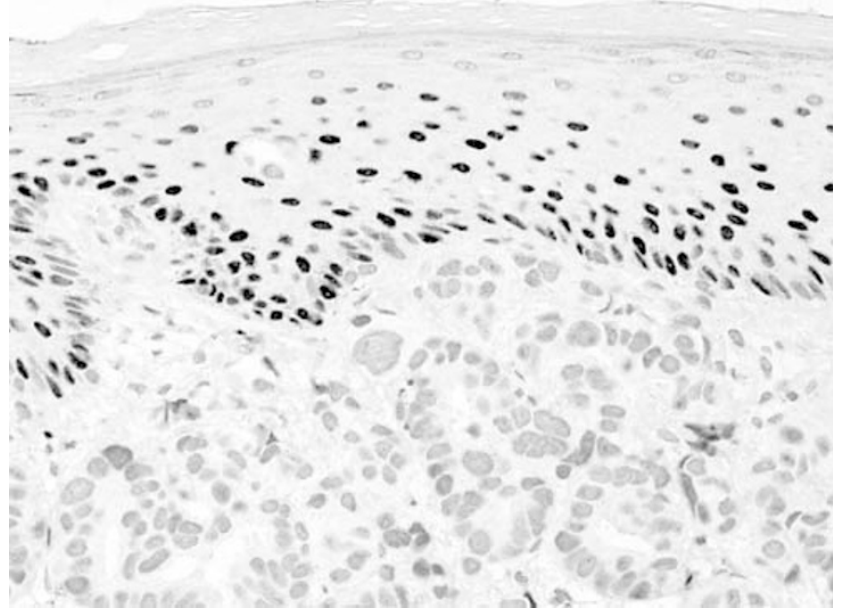

Figure 3 Similar to this case of breast metastatic carcinoma to the skin, none of the metastatic adenocarcinomas expressed p63.

mucinous adenocarcinoma with appendiceal origin), p63 was expressed in less than $5 \%$ of the neoplastic cells. The apical cells revealed mild cytoplasmic staining. 
The differences in p63 immunolabeling between the primary cutaneous adnexal tumors and metastatic adenocarcinomas to the skin were statistically significant $(P<0.001)$.

\section{Discussion}

Analysis of p63 expression in normal tissue and neoplastic conditions has been recently the subject of several basic research studies and also gained a special attention in the applicative pathology literature. It has been reported that $\Delta \mathrm{Np} 63$ isoforms are consistently expressed in the nuclei of the basal cells of the multilayered epithelia, including skin, cervical and vaginal mucosa, urothelium, respiratory tract, as well as by the myoepithelial cells of the breast and sweat glands. ${ }^{1-4,10-15}$ Analysis of p63 expression has been recently incorporated by some in the routine breast pathology diagnosis provided that $p 63$ is a reliable myoepithelial cell marker. ${ }^{14,15}$ Similarly, analysis of p63 expression is used to identify prostatic basal cells in challenging cases. ${ }^{12}$

Reis-Filho et $a l^{11}$ studied the distribution of $p 63$ in 400 neoplastic conditions, the largest case studies to date, and have shown that the vast majority of the adenocarcinomas from breast, prostate, lung, colon and ovary did not expressed p63. Kaufmann et $a l^{20}$ had similar results in their case series of 141 adenocarcinomas, where only 20 cases had variable and mild $p 63$ positivity.

The expression of p63 in normal human epidermis, cutaneous appendages and skin carcinomas has been recently assessed. ${ }^{16-18}$ p63 seems to play a major role in ectodermal development, in the maintenance of the basal cell population of stratified epithelia, and also in the terminal differentiation of epithelia. $^{6-8}$ In vitro studies, using transformed human keratinocytes, have shown that p63 is a nuclear transcription factor that triggers keratinocyte differentiation and is downregulated in terminally differentiated cells. ${ }^{4,5,16}$ Moreover, p63 might block the apoptosis-inducing activity of p53 and thus could help to maintain the proliferative capacity of basal/progenitor cells. ${ }^{5-8}$

However, there is only limited knowledge regarding the pattern of p63 expression in cutaneous adnexal neoplasms, including adenocarcinomas. The potential use of this marker as diagnostic tool in distinguishing between primary vs metastatic cutaneous carcinomas is not fully investigated.

Our study confirmed and expanded the results of previously published studies, namely constant p63 expression in the basal and suprabasal cells of normal epidermis and cutaneous appendages, including basal/myoepithelial cells of the eccrine and apocrine sweat glands.

In the hair follicle-derived tumors, the cell components originating from the outer root sheath cells or matrix cells expressed p63. All trichoepitheliomas exhibited $p 63$ positivity. The small cords and islands of basaloid cells of desmoplastic trichoepitheliomas were strongly highlighted by $p 63$ labeling. The benign tumors derived from the sweat glands of either eccrine or apocrine origin (syringomas, eccrine poromas and benign tubular adenoma) also exhibited p63. The tricholemmal carcinomas, derived from outer root sheath cells, preserved $p 63$ expression. In the microcystic adnexal carcinomas, the islands and strands of basaloid cells expressed p63, in contrast with the keratinous cysts. The eccrine adenocarcinomas did also exhibit strong p63 positivity. Only in the case of mucinous adenocarcinoma p63 was expressed in less than $25 \%$ of the cells.

None of the metastatic carcinomas exhibited p63 positivity $(P<0.001)$ and this raises the hypothesis that this marker expression might be used for distinguishing metastatic adenocarcinomas to the skin from primary cutaneous adnexal neoplasms.

In summary, p63 is consistently expressed in a wide variety of epidermal appendages thus suggesting the participation of basal/myoepithelial cells in the ontogenesis of these tumors. The $p 63$ expression is a sensitive and specific marker for benign or malignant adnexal tumors. Moreover, the analysis of p63 expression may help in the differential diagnosis of primary adnexal carcinomas, including adenocarcinomas vs metastatic cutaneous adenocarcinomas.

\section{References}

1 Yang A, Kaghad M, Wang Y, et al. p63, a p53 homolog at 3q27-29 encodes multiple products with transactivating, death-inducing, and dominant-negative activities. Mol Cell 1988;2:305-316.

2 Yang A, McLeon F. p63 and p73: p53 mimics, menaces and more. Nat Rev Mol Cel Biol 2000;3:199-207.

3 Yang A, Kaghad M, Caput D, et al. On the shoulders of giants: $p 63, p 73$ and the rise of $p 53$. Trends Genet 2002;18:90-95.

4 Levrero M, De Laurenzi V, Constanzo A, et al. The p53/ $p 63 / p 73$ family of transcription factors: overlapping and distinct functions. J Cell Sci 2000;113:1661-1670.

5 Pellegrini G, Dellambra E, Golisano O, et al. p63 identifies keratinocyte stem cells. Proc Natl Acad Sci USA 2001;98:3156-3161.

6 Mills AA, Zheng B, Wang XJ, et al. p63 is a p53 homologue required for limb and epidermal morphogenesis. Nature 1999;398:708-713.

7 Yang A, Schweitzer R, Sun D. p63 is essential for regenerative proliferation in limb, craniofacial and epithelial development. Nature 1999;398:714-718.

8 Parsa R, Yang A, McLeon F, et al. Association of p63 in proliferative potential in normal and neoplastic human keratinocytes. J Invest Dermatol 1999;113:1099-1105.

9 Celli J, Duijf P, Hamel BC. Heterozygous germ cell mutations in the p53 homolog p63 are the cause of ECC syndrome. Cell 1999;99:143-147.

10 Di Como CJ, Urist MJ, Babayan I, et al. p63 expression profiles in normal and tumor tissues. Clin Cancer Res 2002;8:494-501. 
11 Reis-Filho JS, Simpson PT, Martins A, et al. Distribution of $p 63$, cytokeratins $4 / 6$ and cytokeratin 14 and 51 in normal and 400 neoplastic human tissue samples using TARP-4 multi-tumor tissue microarray. Virch Arch 2003;443:122-132.

12 Signoretti S, Waltregny D, Dilks J. p63 is a prostate basal cell marker and is required for prostate development. Am J Pathol 2000;157:1769-1773.

13 Glickman JN, Yang A, Shahsafei A, et al. Expression of p53-related protein p63 in the gastrointestinal tract and in esophageal metaplastic and neoplastic disorders. Hum Pathol 2001;32:1157-1165.

14 Barbareschi M, Pecciarini L, Cangi MG. p63, a p53 homologue, is a selective nuclear marker of myoepithelial cells of human breast. Am J Surg Pathol 2002;25:1054-1062.

15 Reis-Filho JS, Schmitt FC. Taking advantage of basic research: p63 is a reliable myoepithelial and stem cell marker. Adv Anat Pathol 2002;9:280-289.
16 Reis-Filho JS, Torio B, Albergaria A, et al. p63 expression in normal skin and usual cutaneous carcinomas. J Cutan Pathol 2002;29:517-523.

17 Tsujita-Kyutoku M, Kuichi K, Danbara N, et al. p63 expression in normal human epidermis and epidermal appendages and their tumors. J Cutan Pathol 2003;30:11-17.

18 Qureshi HS, Ormsby AH, Lee MW, et al. The diagnostic utility of $p 63, \mathrm{CK} 5 / 6$, CK7, and CK20 in distinguishing primary cutaneous adnexal neoplasms from metastatic carcinomas. J Cutan Pathol 2004;31:145-152.

19 Ivan D, Diwan AH, Esteva FJ, et al. Expression of cell cycle inhibitor $p 27^{K i p 1}$ and its inactivator Jab1 in melanocytic lesions. Mod Pathol 2004;17:811-818.

20 Kaufmann O, Fietze E, Mengs J, et al. Value of p63 and cytokeratin 5/6 as immunohistochemical markers for the differential diagnosis of poorly differentiated and undifferentiated carcinomas. Am J Clin Pathol 2001;116:823-830. 